\title{
Review of: "Genetic basis and adaptation trajectory of soybean from its temperate origin to tropics"
}

Kenji Fujino

Potential competing interests: The author(s) declared that no potential competing interests exist.

The ms focused on the adaptability of soybean, temperate to tropic region. Experimental design was well. The results were clear. This is very interesting on crop adaptability on molecular aspects. Very beautiful and excellent. This is valuable. There were some points to be hard the understanding for the readers.

Major

1. The authors concluded on Tof16 and J. However, only Tof16 was detected in GWAS. I think this is the limitation of GWAS procedure. Please add the reason. This is not on the experimental results. Genes for the controlling flowering time could drive the adaptability. The were possibility to control of the adaptability to tropics than Tof16 and J?

2. Fig. 5. The changes of the allele were drawn in the horizontal. However, temperate-to-tropics is vertical. The author should make the figure in vertical. There were Tof16 $\mathrm{j}$ and tof16 $\mathrm{J}$ in natural variation? The combination of tof16 $\mathrm{j}$ is real or next genotype? Crop breeding programs would join both alleles to stable production in tropics.

Minor

1. The expression of "the mutation of the genes" was unclear. This was both genes or single?

2. Fig. 2. This was complexity. There were many allele combinations. This was depended on the number of alleles used in the study. Simpler figure would make the reader help to understand. You could focus on the limited genotype?

3. Fig. 3h, i. These were interesting. But it was hard to read the figure.

4. Fig. 4. Can you join the evolutional network of Tof16 and J? If the genes were under selection, you should focus on network of both genes. 